\section{Fish scales for human origins}

SIR-Previous morphological analyses using hennigian cladistic approaches had suggested that Lake Victoria's approximately 200 species of haplochromine cichlid fishes had a polyphyletic origin'. Despite marked morphological diversity, Lake Victoria's cichlid fish flock may be monophyletic and may have arisen from one ancestral species in fewer than a million years ${ }^{2}$. Although the descendant forms have become adaptively diverse, they appear to exhibit less genetic variation in mitochondrial DNA than does the human species. I would like to suggest that the new data might help to resolve some morphological and molecular problems concerning the origin of modern humans.

Many palaeoanthropologists have held that there was insufficient time to transform the skeletal features diagnostic of more archaic European human populations into those of anatomically modern Homo sapiens, requiring instead an invasion of evolutionarily more advanced populations from outside the region ${ }^{3}$. But early anatomically modern $H$. sapiens populations were less divergent morphologically from late $H$. erectus or other archaic Homo populations than the cichlid Ptyochromis sauvagei is from Astatotilapia piceatus ${ }^{+}$.

The example of Lake Victoria also provides an opportunity to assess the timespans needed for morphological transitions. In nature, the timing of speciation events must be highly irregular and could be influenced by generation lengths, but a few simplifying assumptions might be helpful. Given an outer boundary of up to a million years for the radiation of the Lake Victoria fishes ${ }^{2}$ and assuming an (unlikely) sequential, arithmetic mode of divergence, one new fish species would appear about every 5,000 years. If we use instead a geometric model of speciation, eight rounds of binary population subdivision (occurring approximately once every 125,000 years) could produce say 200 morphologically divergent populations over the same million-year timespan. Alternatively, if all the population fissioning occurred over only 200,000 years, the corresponding estimates for the intervals between splits would be 1,000 years and 25,000 years, respectively.

The longest estimate of about 125,000 years corresponds to several reliable estimates for the approximate duration of Neanderthals in the European fossil record ${ }^{5}$. The shorter estimates of 1,000 to 5,000 years are less than the limits of resolution of various dating methods used to assess the relative temporal relationships of Upper Palaeolithic hominid populations, suggesting that it would be virtually impossible to distinguish between phyletic transformation and replacement.

Finally, what is the significance of the fact that genetic variation is lesser in fishes than among humans? If the fishes all arose within the 250,000 - to 750,000 -year timespan estimated for the existence of Lake Victoria yet exhibit less mitochondrial DNA variation than do humans, assumption of a constant rate of divergence of this DNA would require that the differentiation of humans in major geographical regions of the world must have occurred more than 250,000 to 750,000 years ago. Such a suggestion is consistent with a single expansion of humans out of Africa at about the $H$. erectus level, with continuity through present regional populations, rather than replacement of archaic human populations outside Africa by a second and more recent wave of anatomically modern humans.

It would be possible to escape the temporal constraints of this inference by assuming that mitochondrial DNA divergence rates are inconstant. Then, as I have observed elsewhere ${ }^{6}$, all simple bets are off and it must be concluded that mitochondrial DNA reveals as little about the timing of modern human origins as it does about the morphological transformations visible in the fossil evidence.

Institute of Molecular Evolutionary

Genetics,

The Pennsylvania State University,

University Park

Pennsylvania 16802, USA

1. Greenwood, P.H. The Haplochromine Fishes of the East African Lakes (Cornell Univ. Press, 1981).

Meyer, A. Kor T Nature 347, 550-553 (1990)

3. Spencer, F. in The Origins of Modern Humans (eds Smith F.H. \& Spencer, F.) 1-49 (Liss, New York, 1984).

. Avise, J.C. Nature 347, 512-513 (1990).

. Kline, R.G. The Human Career (Univ. Chicago Press, 1989)

Eckhardt, R.B. Hum. Evol. 4, 317-319 (1989)

\section{Survival test}

SIR-C.H. Daugherty et al. (Nature 347, $177-179 ; 1990$ ) and R. M. May in his News and Views article on page 129 of the same issue draw attention to a case where the absence of taxonomic recognition has allowed extinction of one subspecies and reduced the likelihood that another will ultimately survive. The authors also point out that there is a need for adequate collections and for an intelligent programme of protection incorporating continuous sampling.

Until a little more than 10 years ago, the New Zealand Wildlife Department made it difficult to obtain specimens of the tuatara for most kinds of biological research; individuals were made available mainly for exhibition in zoos or for specific research projects. Sampling merely for anatomy and taxonomy was apparently not among these. I remember being shocked when in 1979 I saw the limited amount of very old and poorly preserved material in the Wellington Museum. As Stephen's Island, less than 100 miles away, contained a population estimated at more than 100,000 specimens, it seemed desirable to have at least enough material in the museum for characterizing one population so that smaller samples might be compared to this. I am glad that the continuing efforts of many people have now permitted an improved level of sampling.

The lesson remains that protectionist impulses that limit or stop sampling do not automatically result in preservation of the species. This is certainly critical in the many parts of the world where forest, game and wildlife departments impose unrealistic bag limits on relatively common but poorly known species.

The second point is that we need to continue the maintenance of reference collections, staffed by taxonomists skilled in various techniques, now more than ever. Specialists can evaluate, add to their holdings and advise agencies regarding the most economic investment of limited money. There is a lesson here for the fate of the Natural History Museum in London, because it is said that its effort is to be diverted to projects that do not require reference collections and do not ultimately contribute to the maintenance and protection of our shrinking global diversity.

\section{Department of Biology,}

The University of Michigan,

Ann Arbor,

Michigan 48109, USA

\section{Earlier light}

SIR-In their report on the use of azobenzene derivatives as the basis of photochemical information-storage devices', Liu et al. made no reference to earlier work by myself and N. Doddapaneni ${ }^{2}$. In that work, we also studied the electrochemical behaviour of cis and trans azobenzenes, their interconversion under light irradiation and their electrochemical reduction to hydroazobenzenes.

This study, together with our related work on polycyclic aromatic quinones was undertaken with the explicit objective of developing organic systems capable of storing and later releasing light energy.

Department of Chemistry,

G. KLOPMAN

\section{Case Western Reserve University,}

Cleveland,

Ohio 44106, USA

\footnotetext{
1. Liu, Z. F., Hashimoto, K. \& Fujisnima, A. Nature 347 $658-660$ (1990)

2. Kopman, G. \& Doddapaneni, N. J. phys. Chem. 78 18251828 (1974)

Klopman, G. \& Doddapaneni, N. J. phys. Chem. 78 , 18201824 (1974)
} 